\title{
Disappearance of mtDNA During Mating of the True Slime Mold Didymium iridis
}

\author{
Yohsuke Moriyama, Kie Itoh, Hideo Nomura and Shigeyuki Kawano* \\ Department of Integrated Biosciences, Graduate School of Frontier Sciences, University of \\ Tokyo, Bldg. FSB-601, 5-1-5 Kashiwanoha, Kashiwa, Chiba 277-8562, Japan
}

Received May 1, 2009; accepted June 29, 2009

\begin{abstract}
Summary The active, selective digestion of mitochondrial DNA (mtDNA) from 1 parent in the zygote is a possible molecular mechanism for the uniparental inheritance of mitochondria, but direct evidence has been observed in few species. In this study, we observed the behavior of mitochondria and mtDNA during mating of the myxamoeba of the true slime mold Didymium iridis. To show the selective digestion of mtDNA in the zygote, 2 myxamoebal strains of $D$. iridis were crossed, and the changes of mitochondria and mtDNA were observed over time by phase-contrast observation using alkaline fixation method and DAPI staining. Each myxamoeba of $D$. iridis contained about 30 mitochondria, and the zygote had about 60 . Each mitochondrion contains rod-shaped mtDNA. About $4.5 \mathrm{~h}$ after mating, the fluorescence of mtDNA in about 30 mitochondria decreased simultaneously to give small spots, and then disappeared completely by $5 \mathrm{~h}$ after mating. In contrast, the mtDNA in the other 30 mitochondria and all of the mitochondrial sheaths remained unchanged. This is the fourth microscopic report that shows selective mtDNA disappearance. The rapid, selective disappearance of mtDNA observed in D. iridis is likely the result of selective digestion of mtDNA from 1 parent, as in other known cases of mtDNA disappearance.
\end{abstract}

Key words Isogamous mating, Mitochondrial transmission genetics, mtDNA, Myxomycetes, Uniparental inheritance.

Uniparental inheritance appears to be a common feature of mitochondrial inheritance (reviewed by Birky 1995, 2001). In oogamous and anisogamous species, uniparental inheritance of mitochondria has been attributed to the small number of mitochondria in the male gamete. Although fertilized eggs are heteroplasmic (i.e., they contain mitochondria from both parents), the small population of mitochondria derived from the male gamete segregates rapidly after repeated cell division. Consequently, most cells are thought to contain mitochondria from the female parent (Ankel-Simons and Cummins 1996). However, the idea of segregation of parental mtDNA has recently been challenged. Several studies have reported that sperm mitochondria are destructed in the mammalian fertilized egg (Sutovsky et al. 1999, 2000, 2003). Meanwhile, the selective digestion of mtDNA has been reported before the destruction of mitochondria in the zygote of the true slime mold Physarum polycephalum (Moriyama and Kawano 2003). About $3.5 \mathrm{~h}$ after mating, the mtDNA in half of the mitochondria disappeared synchronously. The mtDNA of the parental strains was polymorphic, and the differences could be distinguished by PCR. In that report, a single cell was isolated under a microscope using a micromanipulator, and detection of eparental mtDNA was attempted to detect by PCR. PCR analysis revealed that the loss of mtDNA from 1 parent coincided with the disappearance of mtDNA in the zygote. This rapid, selective disappearance of mtDNA could promote the uniparental inheritance of mitochondria in P. polycephalum. After the disappearance of mtDNA, mitochondria that do not contain mtDNA were gradually eliminated by $60 \mathrm{~h}$ after mating.

\footnotetext{
*Corresponding author, e-mail: kawano@k.u-tokyo.ac.jp
} 
The active disappearance of organelle DNA has been originally reported in chloroplast inheritance. Precise microscopic observation revealed that the chloroplast DNA (cpDNA) derived from the male $\left(\mathrm{mt}^{-}\right)$parent disappears after zygote formation in the isogamous green algae Chlamydomonas reinhardtii (Kuroiwa et al. 1982, Nishimura et al. 1999). But there are few reports about direct observation of organelle DNA disappearance in the zygote. In the case of mitochondria, there are 3 reports that show selective disappearance of mtDNA in the zygote (Myxomycetes: Moriyama and Kawano 2003, Fish: Nishimura et al. 2006, Green algae: Aoyama et al. 2006; Table 1). In this study, we observed the behavior of mtDNA during the mating of Myxomycete $D$. iridis, to investigate that selective disappearance of mtDNA is a conserved mechanism for the uniparental inheritance of mitochondria, at least in Myxomycetes. The inheritance mode of mtDNA has already been reported in D. iridis, and it is strictly uniparental (Silliker and Collins 1988, Silliker et al. 2002, Sheer and Silliker 2006). The life cycle of D. iridis includes 2 distinct vegetative forms: the haploid myxamoeba and the diploid plasmodium. The haploid myxamoebae act as isogametes; individuals of different mating types pair and fuse to form diploid zygotes that develop into macroscopic, diploid plasmodia after repeated mitotic cycles without cell division. This precludes mitochondrial segregation by means of cell division as a mechanism for establishing homoplasmy, a homogeneous organelle population. Further, the multiple allelic mating systems common in heterothallic Myxomycetes (Betterley and Collins 1983, Kawano et al. 1987, Kawano and Kuroiwa 1989) mean that mitochondrial donor and recipient strains cannot be simply designated by mating type as would be possible in a binary mating system (Hurst 1992, Hurst and Hamilton 1992). Despite these features, uniparental inheritance occurs in D. iridis (Silliker and Collins 1988, Silliker et al. 2002, Sheer and Silliker 2006). Therefore, an active mechanism that eliminates mitochondria or mtDNA from 1 parent must be involved. In an attempt to understand the universal mechanism of uniparental inheritance, we observed the dynamics of mitochondria and mtDNA throughout mating of the true slime mold $D$. iridis. Consequently, selective disappearance of mtDNA was also observed in D. iridis.

\section{Materials and methods}

\section{Strains and culture}

Myxamoebal strains of Didymium iridis H7 and P4 (\#24464 and \#24465) were purchased from The American Type Culture Collection. Myxamoebae were cultured on PGY plates $(0.5 \%$ glucose, $0.05 \%$ yeast extract, $2 \mathrm{mM} \mathrm{MgSO}_{4}$, and $1.5 \%$ agar in $25 \mathrm{mM} \mathrm{K}_{3} \mathrm{PO}_{4}$ buffer, $\left.\mathrm{pH} 6.6\right)$ at $23^{\circ} \mathrm{C}$ with live bacteria (Klebsiella aerogenes) for food. Zygote formation was induced on mating plates (1.5\% agar) at $23^{\circ} \mathrm{C}$ by mixing $\mathrm{H} 7$ and $\mathrm{P} 4.2$ strains were mixed equally, and the suspensions were put onto the plate at 2000 cells $/ \mathrm{mm}^{2}$.

\section{Microscopic observation}

The myxamoebae and zygotes were fixed with small drops of formaldehyde solution adjusted to the alkalinity [ $8 \%$ formaldehyde in $10 \times \mathrm{PBS}$ ( $\mathrm{pH} 11.0$ ) containing $0.01 \%$ Tween 20 ] on the $1.5 \%$ agar plate. DNA was stained with 4',6-diamidino-2-phenylindole (DAPI), which was resolved in $1 \mu \mathrm{g} / \mathrm{ml}$ DAPI in TAN buffer [20 mM Tris-HCl (pH 7.6), $0.5 \mathrm{mM}$ EDTA, $7 \mathrm{mM} \beta$-mercaptoethanol, $1.2 \mathrm{mM}$ spermidine], and a coverslip was placed over the stained sample (Moriyama and Kawano 2003). Photographs were taken with a BX62 epifluorescence microscope (Olympus, JAPAN) equipped with a C4742 CCD camera (Hamamatsu Photonics Co., Japan) and an Aquacosmos system (Hamamatsu Photonics Co., Japan). 
Table 1. Microscopic observations of mtDNA degradation in the literature

\begin{tabular}{|c|c|c|c|c|}
\hline \multirow{2}{*}{ Common name } & \multirow{2}{*}{ Species } & \multicolumn{2}{|c|}{ Observation method } & \multirow{2}{*}{ Reference } \\
\hline & & mtDNA & Mitochondria & \\
\hline True slime mold & Physarum polycephalum & DAPI & PC* observation & Moriyama and Kawano, 2003 \\
\hline Medaka & Oryzias latipes & SYBR Green I & MitoTracker & Nishimura et al., 2006 \\
\hline Green algae & Chlamydomonas smithii & FISH, DAPI & - & Aoyama et al., 2006 \\
\hline True slime mold & Didymium iridis & DAPI & PC observation & This study \\
\hline
\end{tabular}

* PC: phase-contrast.

\section{Results}

Active digestion of mitochondria from 1 parent in the zygote has been observed in 3 species (Table 1).

Myxamoebae are uninucleate cells that act as isogametes in crosses. To observe the mating process, we attempted crosses using several myxamoebal strains of $D$. iridis, and examined the mating efficiency after mixing each pair of strains. Since the combination of H7 and P4 mated most frequently, we used those 2 strains. Phase-contrast observations of myxamoebae clearly revealed the elliptical mitochondria. After DAPI staining, cell nuclei and mtDNA emitted bright blue-white fluorescence. Each $\mathrm{H} 7$ and P4 myxamoeba contained about 30 mitochondria before mating, and each mitochondrion contained a long rod-shaped mtDNA at its center (Fig. 1A). The shapes of H7 and $\mathrm{P} 4$ cells were very similar. In our serial observations of mating, cell fusion occurred $2 \mathrm{~h}$ after mixing the 2 strains. Immediately, the full complement of about 60 parental mitochondria mixed (Fig. 1B). The fused cells formed a uninucleate zygote via nuclear fusion, about $2.5 \mathrm{~h}$ after mating. A fluorescent mtDNA in some mitochondria persisted in every mitochondrion until this stage. However, $2 \mathrm{~h}$ after nuclear fusion, the mtDNA fluorescence diminished synchronously to small spots, regardless of the position of the mitochondria in the zygote (Fig. 1C). Within a further $30 \mathrm{~min}$, the mtDNA fluorescence in these mitochondria disappeared completely (Fig. 1D). Fig. 1E-H shows the precise manner of mtDNA disappearance. A long, rod-shaped mtDNA was present in each mitochondrion of the myxamoebae (Fig. 1E). After cell fusion, the shapes of the mitochondria and mtDNA were consistent with the myxamoebal stage (Fig. 1F). In half, the mtDNA disappeared, starting from both ends of the mtDNA about $2 \mathrm{~h}$ after nuclear fusion (Fig. 1G). The fluorescence of each mtDNA grew fainter and rapidly reduced to a single small spot. This spot was consistently located at the center of the mitochondrion, and it disappeared completely without any other apparent major changes of the mitochondrial shape (Fig. $1 \mathrm{H})$. At this time, about half of the mitochondria in each zygote lacked an mtDNA, although the shape of the mitochondria matched that in the myxamoebal stage (Figs. 1D, H). According to this observation, the disappearance of mtDNA began $4.5 \mathrm{~h}$ after mating, and was completed by $5 \mathrm{~h}$ after mating.

\section{Discussion}

We have previously revealed that the rapid, selective digestion of mtDNA promotes the uniparental inheritance of mitochondria in the true slime mold $P$. polycephalum (Moriyama and Kawano 2003). Microscopic and molecular analysis of a single cell showed that the disappearance of mtDNA coincided with the uniparental inheritance of mitochondria; after mtDNA disappearance, PCR analysis could detect the mtDNA from only 1 of the 2 parents. The mitochondria that did not contain mtDNA were gradually destroyed in the zygote of $P$. polycephalum. The same result was obtained in the inheritance of cpDNA in the green alga C. reinhardtii (Nishimura et al. 2002). 

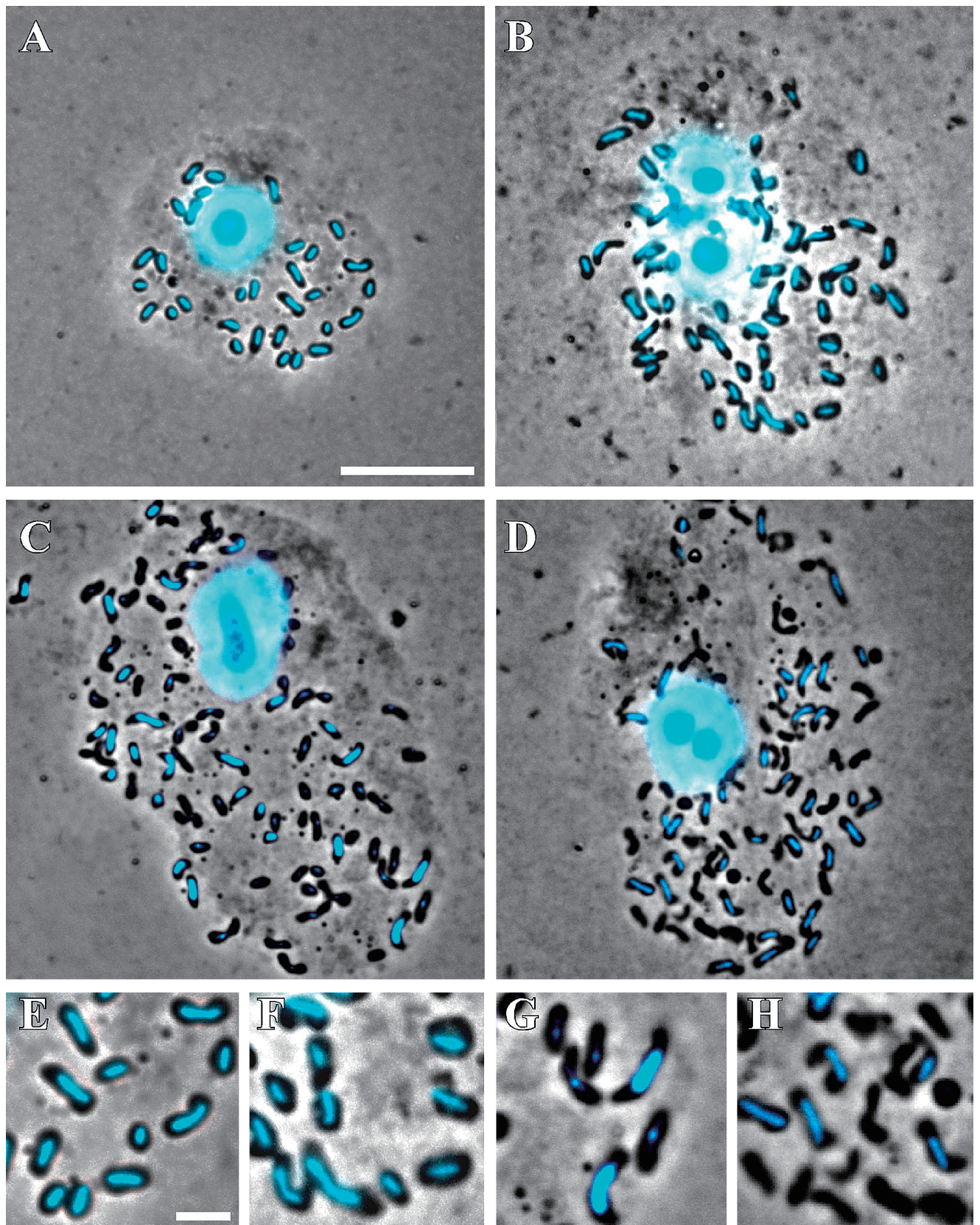

Fig. 1. Disappearance of mtDNA during zygote formation of D. iridis. A-H, Merged phase-contrast and fluorescence microscopy images. A-D, The mating process of $D$. iridis. E-H, High magnification of A-D to show the precise manner of mtDNA disappearance. A, E. representative myxamoeba $(\mathrm{H} 7)$. B, F. fused cell $(\mathrm{H} 7 \times \mathrm{P} 4)$. C, G. uninucleate zygote $(\mathrm{H} 7 \times \mathrm{P} 4)$ of $4.5 \mathrm{~h}$ after mating. D, H. uninucleate zygote $(\mathrm{H} 7 \times \mathrm{P} 4)$ of $5 \mathrm{~h}$ after mating. Scale bars: $\mathrm{A}-\mathrm{D}, 10 \mu \mathrm{m} . \mathrm{E}-\mathrm{H}, 2 \mu \mathrm{m}$.

If mtDNA from 1 parent was replicated selectively in the zygote, and the repeated cell division occurred, mtDNA could be inherited uniparentally. However, after the zygote formation, cell division never occurs in the Myxomycete. The number or the shape of mtDNA did not change till just before the disappearance of fluorescent mtDNA. Thus, the selective replication of mtDNA is not 
concerned in uniparental inheritance of mitochondria.

In $D$. iridis, the inheritance mode of mitochondria has been analyzed. The results showed that the inheritance mode of mitochondria is predominantly uniparental (Silliker and Collins 1988; Silliker et al. 2002, Sheer and Silliker 2006). The uniparental inheritance of organelle is a widely conserved phenomenon in many organisms, and as the 2 Myxomycetes $P$. polycephalum and D. iridis are relatively closely related species, it could be expected that they possess the same mechanism for the uniparental inheritance of mitochondria. As a result, our observations show that the mtDNA disappeared selectively in the zygote of $D$. iridis. The earlier study examined the inheritance mode of mtDNA from the plasmodia of 8-12 days after mating (Silliker and Collins 1988, Silliker et al. 2002), but the uniparental inheritance of mitochondria could be completed by a much earlier stage of mating, at most $5 \mathrm{~h}$ after mating. As in . polycephalum, the selective digestion of mtDNA should promote the uniparental inheritance of mitochondria in D. iridis.

In nearly all eukaryotes, mitochondria are inherited uniparentally. However, the mechanism behind uniparental inheritance is unclear. To reveal whether the selective digestion of mtDNA is a universal mechanism for the uniparental inheritance of mitochondria, more observations are needed of the behavior of mitochondria during mating and fertilization in many species. The disappearance of mtDNA has also been demonstrated in higher plants and algae. This disappearance of mtDNA occurs before fertilization in the mature generative cell inside a pollen grain or in the male gamete before fertilization (Kuroiwa and Hori 1986, Corriveau and Coleman 1991, Nagata et al. 1999). By contrast, in $P$. polycephalum and D. iridis, mtDNA disappearance occurs in the zygote after mating. Although mitochondria from both parents were mixed in the zygote cell, mtDNA from the mitochondrial recipient strain disappeared synchronously and completely, while that from the mitochondrial donor strain was completely conserved. Highly selective and active nuclease(s) are likely involved in the selective digestion of mtDNA. Nishimura et al. (2002) had reported the candidate of nuclease that might participate in the digestion of cpDNA from one parent in C. reinhardtii. In $P$. polycephalum, we have also detected a zygote specific nuclease activity that is specifically incorporated into mitochondria just before the digestion of mtDNA (Moriyama et al., 2005). In D. iridis, the disappearance of mtDNA progresses as same manner as in $P$. polycephalum. Forty to eighty copies of mtDNA are organized with several proteins into rod-shaped mt-nucleoid. The ends of the nucleoid are the most accessible position for the nuclease. We think, thus, the manner of mtDNA disappearance could be due to nuclease activity also in $D$. iridis. The zygote stage specific incorporation of nuclease into mitochondria can explain the disappearance of mtDNA from each end of the nucleoid toward the middle. The most effective approach to reveal the mechanism behind the uniparental inheritance of mitochondria might be to find the nuclease during mating.

\section{Acknowledgments}

We especially thank Dr. M. E. Silliker (Department of Biological Sciences, Depaul University) for the generous gift of the myxamoeba strains. We thank Prof. T. Kuroiwa (Research Information Center for Extvemophile, Rikkyo University) for helpful discussions. We also thank Dr. S. Matsunaga (Graduate School of Engineering, University of Osaka) for helpful technical advice. This study was supported by grants for Scientific Research in Priority Areas (no. 13440246 to S. K.) from the Ministry of Education, Culture, Sports, Science, and Technology of Japan.

\section{References}

Ankel-Simons, F. and Cummins, J. M. 1996. Misconceptions about mitochondria and mammalian fertilization: implications for theories on human evolution. Proc. Natl. Acad. Sci. USA 93: 13859-13863.

Aoyama, H., Hagiwara, Y., Misumi, O., Kuroiwa, T. and Nakamura, S. 2006. Complete elimination of maternal mitochon- 
drial DNA during meiosis resulting in the paternal inheritance of the mitochondrial genome in Chlamydomonas species. Protoplasma 228: 231-241.

Betterley. D. A. and Collins, O. R. 1983. Reproductive systems, morphology, and general diversity in Didymium iridis (Myxomycetes). Mycologia 75: 1044-1063.

Birky, C. W. Jr. 1995. Uniparental inheritance of mitochondrial and chloroplast genes: mechanisms and evolution. Proc. Natl. Acad. Sci. USA 92: 11331-11338.

- 2001. The inheritance of genes in mitochondria and chloroplasts: Laws, Mechanisms, and Models. Annu. Rev. Genet. 35: $125-148$.

Corriveau, J. L. and Coleman, A. W. 1991. Monitoring by epifluorescence microscopy of organelle DNA fate during pollen development in five angiosperm species. Dev. Biol. 147: 271-280.

Hurst, L. D. 1992. Intragenomic conflict as an evolutionary force. Proc. R. Soc. Lond. B 248: 135-140.

— and Hamilton, W. 1992. Cytoplasmic fusion and the nature of sexes. Proc. R. Soc. Lond. B 247: 189-194.

Kawano, S., Kuroiwa, T. and Anderson, R. W. 1987. A 3rd multiallelic mating-type locus in Physarum polycephalum. J. Gen. Microbial. 133: 2539-2546.

— and - 1989. Transmission pattern of mitochondrial-DNA during plasmodium formation in Physarum polycephalum. J. Gen. Microbiol. 135: 1559-1566.

Kuroiwa, T., Kawano, S., Nishibayashi, S. and Sato, C. 1982. Epifluorescent microscopic evidence for maternal inheritance of chloroplast DNA. Nature 298: 481-483.

— and Hori, T. 1986. Preferential digestion of male chloroplast nuclei and mitochondrial nuclei during gametogenesis of Bryopsis maxima Okamura. Protoplasma 133: 85-87.

Moriyama, Y. and Kawano, S. 2003. Rapid, selective digestion of mitochondrial DNA in accordance with the matA hierarchy of multiallelic mating-types in the mitochondrial inheritance of Physarum polycephalum. Genetics 164: 963-975.

- Yamazaki, T., Nomura, H., Sasaki, N. and Kawano, S. 2005. Early zygote-specific nuclease in mitochondria of the true slime mold Physarum polycephalum. Curr. Genet. 485: 334-343.

Nagata, N., Saito, C., Sakai, A., Kuroiwa, H. and Kuroiwa, T. 1999. The selective increase or decrease of organellar DNA in generative cells just after pollen mitosis one controls cytoplasmic inheritance. Planta 209: 53-65.

Nishimura, Y., Misumi, O., Matsunaga, S., Higashiyama, T., Yokota, A. and Kuroiwa, T. 1999. The active digestion of uniparental chloroplast DNA in a single zygote of Chlamydomonas reinhardtii is revealed by using the optical tweezers. Proc. Natl. Acad. Sci. USA 96: 12577-12582.

—, - , O., Kato, K., Inada, N., Higashiyama, T., Momoyama, Y. and Kuroiwa, T. 2002. An mt(+) gamete-specific nuclease that targets $\mathrm{mt}(-)$ chloroplasts during sexual reproduction in C. reinhardtii. Genes Dev. 16: 1116-1128.

—, Yoshinari, T., Naruse, K., Yamada, T., Sumi, K., Mitani, H., Higashiyama, T. and Kuroiwa, T. 2006. Active digestion of sperm mitochondrial DNA in single living sperm revealed by optical tweezers. Proc. Natl. Acad. Sci. USA 103: $1382-1387$.

Silliker, M. E. and Collins, O. R. 1988. Non-mendelian inheritance of mitochondrial DNA and ribosomal DNA in the Myxomycete, Didymium iridis. Mol. Gen. Genet. 213: 370-378.

Silliker, M. E., Liles, J. L. and Monroe, J. A. 2002. Patterns of mitochondrial inheritance in the myxogastrid Didymium iridis. Mycologia 94: 939-946.

Sheer, M. A. and Silliker, M. E. 2006. Mitochondrial inheritance patterns in Didymium iridis are not influenced by stage of mating competency. Mycologia 98: 51-56.

Sutovsky. P., Moreno, R. D., Ramalho-Santos, J., Dominko, T., Simerly, C. and Schatten, G. 1999. Ubiquitin tag for sperm mitochondria. Nature 402: 371-372.

,,,,----- and -2000 . Ubiquitinated sperm mitochondria, selective proteolysis, and the regulation of mitochondrial inheritance in mammalian embryos. Biol. Reprod. 63: 582-590.

—, McCauley, T. C., Sutovsky, M. and Day, B. N. 2003. Early degradation of paternal mitochondria in domestic pig (Sus scrofa) is prevented by selective proteasomal inhibitors lactacystin and MG132. Biol. Reprod. 68: 1793-1800. 